\title{
A RESOLUTION OF THE EULER OPERATOR. I
}

\author{
PETER J. OLVER AND CHEHRZAD SHAKIBAN
}

\begin{abstract}
An exact sequence resolving the Euler operator of the calculus of variations for polynomial differential equations in one independent and one dependent variable is described. This resolution provides readily verifiable necessary and sufficient conditions for such a polynomial differential equation to be the Euler equation for some Lagrangian. An explicit construction of the Lagrangian is given.
\end{abstract}

1. The main result. In the calculus of variations the Euler operator $E$ plays a fundamental role. If $I=\int L\left(x, u, u_{x}, u_{x x}, \ldots\right) d x$ is a variational problem with Lagrangian $L$, then the Euler equation $E(L)=0$ forms a necessary condition for an extremal of $I$. Conversely, suppose we are given a polynomial differential equation $P\left(x, u, u_{x}, u_{x x}, \ldots\right)=0$. In this paper we derive a necessary and sufficient condition for this equation to be the Euler equation for some Lagrangian $L$.

Let $R\{u\}$ denote the ordinary differential algebra consisting of all differential polynomials in the dependent variable $u$ and its various derivatives with respect to the independent variable $x$. We distinguish two cases: when the polynomials all have constant coefficients, and when the polynomials have arbitrary smooth functions as coefficients. The results to be described hold equally well in both cases.

For convenience abbreviate $u_{i}=d^{i} u / d x^{i}$. Given a multi-index $I=$ $\left(i_{1}, i_{2}, \ldots, i_{n}\right)$, where $0 \leqslant i_{j} \in \mathbf{Z}$, let $u_{I}$ denote the monomial $u_{i_{1}} u_{i_{2}} \cdots u_{i_{n}}$. If $I=\left(i_{1}, \ldots, i_{n}\right)$, define \#I=n. Let $R_{n}$ be the subspace of $R\{u\}$ spanned by all $n$-monomials; i.e.,

$$
R_{n}=\operatorname{Span}\left\{u_{I}: \# I=n\right\}, \quad n \geqslant 1 .
$$

The (total) derivative, $d / d x$, acting on $R\{u\}$ is the operator

$$
D=\frac{d}{d x}=\frac{\partial}{\partial x}+\sum_{i=0}^{\infty} u_{i+1} \frac{\partial}{\partial u_{i}} .
$$

Similarly, the Euler operator is

$$
E=\sum_{i=0}^{\infty}(-D)^{i} \frac{\partial}{\partial u_{i}}
$$

Define the operator

Received by the editors March 17, 1977 and, in revised form, September 17, 1977.

AMS (MOS) subject classifications (1970). Primary 49A05; Secondary 12H05.

Key words and phrases. Calculus of variations, Euler operator, Lagrangian, differential algebra. 


$$
F=\sum_{i=0}^{\infty}(-D)^{i} u \frac{\partial}{\partial u_{i}}
$$

Let

$$
N=\sum_{i=0}^{\infty} u_{i} \frac{\partial}{\partial u_{i}} .
$$

(The operator $N$, when restricted to $R_{n}$, is just multiplication by $n$.) Note that to apply $N, D, E$ or $F$ to any given polynomial, only finitely many terms in the sum are needed. The main result of this paper is

THEOREM 1. The complex

$$
0 \rightarrow \mathbf{R} \rightarrow R\{u\} \stackrel{D}{\rightarrow} R\{u\} \stackrel{E}{\rightarrow} R\{u\} \stackrel{F-N}{\rightarrow} R\{u\} \stackrel{F+1}{\rightarrow} R\{u\} \stackrel{F-N}{\rightarrow} R\{u\} \stackrel{F+1}{\rightarrow} \ldots
$$

is exact. (Here 1 denotes the identity map.)

This theorem solves a problem posed by $\mathbf{I}$. M. Gel'fand in a series of lectures delivered at Harvard University and MIT in June, 1976. A subsequent paper, in preparation, will generalize Theorem 1 to differential algebras in several independent and dependent variables.

Corollary 2. If $P \in R\{u\}$ is a differential polynomial, then $P=E(L)$ for some Lagrangian $L$ if and only if

$$
F(P)=N(P)
$$

In fact, if $P$ satisfies (4), then the Lagrangian $L$ can be explicitly given as

$$
L=u \cdot(N+1)^{-1} P \text {. }
$$

This can be directly verified as follows:

$$
\begin{aligned}
E(u P) & =\sum_{i=0}^{\infty}(-D)^{i} \frac{\partial}{\partial u_{i}}(u P)=P+\sum_{i=0}^{\infty}(-D)^{i} u \frac{\partial}{\partial u_{i}} P \\
& =P+F(P)=(N+1) P
\end{aligned}
$$

EXAMPLE. Let $L=\frac{1}{2} u_{x x}^{2}-\frac{1}{6} u_{x}^{3}$. Then

$$
P=E(L)=-D\left(-\frac{1}{2} u_{x}^{2}\right)+D^{2}\left(u_{x x}\right)=u_{x} u_{x x}+u_{x x x x} .
$$

Now

$$
F(P)=-D\left(u u_{x x}\right)+D^{2}\left(u u_{x}\right)+D^{4}(u)=2 u_{x} u_{x x}+u_{x x x x}=N P .
$$

Substituting in (5), we have

$$
L^{\prime}=u \cdot(N+1)^{-1} P=\frac{1}{3} u u_{x} u_{x x}+\frac{1}{2} u u_{x x x x}
$$

as another Lagrangian for $P$. Indeed,

$$
L^{\prime}=L+D\left(\frac{1}{2} u u_{x x x}-\frac{1}{2} u_{x} u_{x x}+\frac{1}{6} u u_{x}^{2}\right)
$$

hence $E\left(L^{\prime}\right)=E(L)=P$.

The proof of Theorem 1 will be done here just for the case of constant 
coefficient polynomials. The main tool is a transform due to Gel'fand' and Dikii, which is described in \$2. An analogous proof of the result for polynomials with arbitrary smooth functions as coefficients can be constructed using the Fourier transform; for the sake of brevity this will be omitted.

2. The Gel'fand-Dikii transform. The notation

$$
S^{n}=\bigodot_{*} \mathbf{R}^{n}=\bigoplus_{i=0}^{\infty} \bigodot_{i} \mathbf{R}^{n}, \quad n \geqslant 1,
$$

will be used to denote the symmetric algebra of $\mathbf{R}^{n}$, cf. [2, Chapter 1]. If $\left\{e_{1}, e_{2}, \ldots, e_{n}\right\}$ is a basis of $\mathbf{R}^{n}$, then

$$
\left\{e^{I}=e_{1}^{i_{1}} \odot e_{2}^{i_{2}} \odot \cdots \odot e_{n}^{i_{n}}: I=\left(i_{1}, \ldots, i_{n}\right), 0 \leqslant i_{j} \in Z\right\}
$$

forms a basis of $S^{n}$. Note that if $I=\left(i_{1}, \ldots, i_{n}\right)$ and $J=\left(j_{1}, \ldots, j_{n}\right)$, then $e^{I} \odot e^{J}=e^{I+J}$, where $I+J=\left(i_{1}+j_{1}, \ldots, i_{n}+j_{n}\right)$. Elements $\varphi \in S^{n}$ may be thought of as polynomials in the variables $e_{1}, \ldots, e_{n}$, so we shall write $\varphi=\varphi\left(e_{1}, \ldots, e_{n}\right)$. This will enable us to easily construct transformations of $S^{n}$ by replacing one or more of the arguments $\varphi$ by a new linear combination of the $e_{j}$ 's.

Suppose $\pi$ is a permutation of the integers $1,2, \ldots, n$. There is an induced map $\hat{\pi}: S^{n} \rightarrow S^{n}$ which permutes the basis vectors of $\mathbf{R}^{n}$ :

$$
\hat{\pi}\left[\varphi\left(e_{1}, \ldots, e_{n}\right)\right]=\varphi\left(e_{\pi(1)}, e_{\pi(2)}, \ldots, e_{\pi(n)}\right), \quad \varphi \in S^{n} .
$$

Let

$$
\sigma=\frac{1}{n !} \sum_{\pi} \hat{\pi}: S^{n} \rightarrow S^{n}
$$

be the symmetrizing map, the sum being taken over all permutations $\pi$ of the integers $1,2, \ldots, n$. Note that $\sigma$ is a projection, i.e., $\sigma \circ \sigma=\sigma$. Let $S_{0}^{n}=$ $\sigma\left(S^{n}\right)$. Note that $\psi \in S_{0}^{n}$ if and only if $\hat{\pi}(\psi)=\psi$ for all permutations $\pi$. Let

$$
S^{*}=\bigoplus_{n=0}^{\infty} S^{n}, \quad S_{0}^{*}=\bigoplus_{n=0}^{\infty} S_{0}^{n}
$$

(By convention, $S^{0}=S_{0}^{0}=\mathbf{R}$.)

Definition 3. The Gel'fand-Dikĭ transform is the linear map $\mathscr{F}: R\{u\} \rightarrow$ $S^{*}$ such that for any multi-index $I, \mathcal{F}\left(u_{I}\right)=\sigma\left(e^{I}\right)$.

EXAMPLES. For the differential polynomial $P=u_{x x} \in R_{1}$ the Gel'fandDikiı transform is $\mathscr{F}(P)=e_{1}^{2} \in S^{1}$. For the differential polynomial

$$
Q=u_{x} u_{x x x}+3 u u_{x x} \in R_{2}
$$

the transform is

$$
\mathcal{F}(Q)=\frac{1}{2}\left[e_{1} \odot e_{2}^{3}+e_{1}^{3} \odot e_{2}+3 e_{1}^{2}+3 e_{2}^{2}\right] \in S^{2} .
$$

Finally, the transform of a constant monomial $c$ is just the same constant considered as an element of $S^{0}=\mathbf{R}$.

THEOREM 4 [1]. The Gel'fand-Dikii transform gives a linear isomorphism $\mathcal{F}$ : $R_{n} \stackrel{\sim}{\rightarrow} S_{0}^{n}, n \geqslant 0$. 
If $P \in R\{u\}$ is a differential polynomial, then we shall denote $\mathscr{F}(P)=\hat{P}$, in analogy with the Fourier transform of classical analysis. Similarly, if $\Phi$ : $R\{u\} \rightarrow R\{u\}$ is a map, then $\hat{\Phi}: S_{0}^{*} \rightarrow S_{0}^{*}$ will be the unique map satisfying $\hat{\Phi}(\hat{P})=\widehat{\Phi(P)}, P \in R\{u\}$.

LEMMA 5. For $\varphi \in S_{0}^{n}$,

$$
\begin{aligned}
\hat{D}(\varphi) & =\left(e_{1}+e_{2}+\cdots+e_{n}\right) \odot \varphi \\
\hat{E}\left[\varphi\left(e_{1}, \ldots, e_{n}\right)\right] & =n \varphi\left(e_{1}, \ldots, e_{n-1},-e_{1}-e_{2}-\cdots-e_{n-1}\right) .
\end{aligned}
$$

COROLlaRY 6 [1]. The sequence

$$
0 \rightarrow \mathbf{R} \rightarrow S_{0}^{*} \stackrel{\hat{D}}{\rightarrow} S_{0}^{*} \stackrel{\hat{E}}{\rightarrow} S_{0}^{*}
$$

is exact.

Note that by Theorem 4, the exactness of the transformed sequence implies the exactness of the original sequence; hence we have shown that

$$
0 \rightarrow \mathbf{R} \rightarrow R\{u\} \stackrel{D}{\rightarrow} R\{u\} \stackrel{E}{\rightarrow} R\{u\}
$$

is exact.

3. The constant coefficient case. Define the maps

$$
\hat{F}_{j}: S^{n} \rightarrow S^{n}, \quad 1 \leqslant j \leqslant n,
$$

by

$$
\hat{F}_{j}\left[\varphi\left(e_{1}, \ldots, e_{n}\right)\right]=\varphi\left(e_{1}, \ldots, e_{j-1},-e_{1}-e_{2}-\ldots-e_{n}, e_{j+1}, \ldots, e_{n}\right) .
$$

LEMMA 7. If $F$ is the operator defined by (3), then on $S_{0}^{n}$,

$$
\hat{F}=\sum_{j=1}^{n} \hat{F}_{j}
$$

Proof. Suppose $I=\left(i_{1}, \ldots, i_{n}\right)$. Then

$$
F\left(u_{I}\right)=\sum_{j=1}^{n}(-D)^{i_{j}} u_{l_{j}},
$$

where $I_{j}=\left(i_{1}, \ldots, i_{j-1}, 0, i_{j+1}, \ldots, i_{n}\right)$. Therefore

$$
\begin{aligned}
\hat{F}\left[\sigma\left(e^{I}\right)\right] & =\mathscr{F}\left[F\left(u_{l}\right)\right]=\sigma \sum_{j=1}^{n}\left(-e_{1}-e_{2}-\cdots-e_{n}\right)^{i_{j}} \odot e^{I_{j}} \\
& =\sigma \sum_{j=1}^{n} \hat{F}_{j}\left(e^{I}\right)=\sigma \sum_{j=1}^{n} \hat{F}_{j}\left[\sigma\left(e^{I}\right)\right] .
\end{aligned}
$$

Next note that if $\pi_{i j}$ is the transposition interchanging $i$ and $j$, then, on $S_{0}^{n}$,

$$
\hat{\pi}_{i j} \circ \hat{F}_{j}=\hat{F}_{i}, \quad \hat{\pi}_{i j} \circ \hat{F}_{k}=\hat{F}_{k}, \quad i, j \neq k .
$$

Therefore, if $\pi$ is any permutation of $1,2, \ldots, n$ with $\pi(j)=k$, then $\hat{\pi} \circ \hat{F}_{j}$ $=\hat{F}_{k}$ on $S_{0}^{n}$. We conclude that

$$
\hat{F}=\sigma \circ \sum \hat{F}_{j}=\sum \hat{F}_{j} .
$$


LEMMA 8. On $S_{0}^{n}$,

$$
\hat{F}_{j} \circ \hat{F}_{k}=\hat{F}_{k}, \quad j \neq k, \quad \hat{F}_{j} \circ \hat{F}_{j}=1 .
$$

Proof. Let $\varphi \in S_{0}^{n}$. Then

$$
\begin{aligned}
& \hat{F}_{j} \circ \hat{F}_{j}\left[\varphi\left(e_{1}, \ldots, e_{n}\right)\right] \\
&=\hat{F}_{j}\left[\varphi\left(e_{1}, \ldots, e_{j-1},-e_{1}-\ldots-e_{n}, e_{j+1}, \ldots, e_{n}\right)\right] \\
&=\varphi\left(e_{1}, \ldots, e_{j-1}, e_{j}, e_{j+1}, \ldots, e_{n}\right) .
\end{aligned}
$$

Also

$$
\begin{aligned}
& \hat{F}_{j} \circ \hat{F}_{k}\left[\varphi\left(e_{1}, \ldots, e_{n}\right)\right]=\hat{F}_{j}\left[\varphi\left(e_{1}, \ldots,-e_{1}, \ldots . . .-e_{n}, \ldots, e_{n}\right)\right] \\
& =\varphi\left(e_{1}, \ldots,-e_{1}-. \stackrel{j}{\cdot}-e_{n}, \ldots, \stackrel{k}{e}_{j}, \ldots, e_{n}\right) \\
& =\varphi\left(e_{1}, \ldots,-e_{1}-\ldots k-e_{n}, \ldots, e_{n}\right) \text {, }
\end{aligned}
$$

since $\varphi \in \operatorname{im} \sigma$.

Corollary 9. On $S_{0}^{n}, \hat{F} \circ \hat{F}=(n-1) \hat{F}+n$.

Proof.

$$
\hat{F} \circ \hat{F}=\sum_{i, j=1}^{n} \hat{F}_{i} \circ \hat{F}_{j}=\sum_{\substack{i, j=1 \\ i \neq j}}^{n} \hat{F}_{j}+n=(n-1) \hat{F}+n .
$$

Corollary 10. The sequences

$$
S_{0}^{n} \stackrel{\hat{F}-n}{\rightarrow} S_{0}^{n} \stackrel{\hat{F}+1}{\rightarrow} S_{0}^{n}, \quad S_{0}^{n} \stackrel{\hat{F}+1}{\rightarrow} S_{0}^{n} \stackrel{\hat{F}-n}{\rightarrow} S_{0}^{n}
$$

are exact.

Proof. By Corollary 9, $(\hat{F}+1)(\hat{F}-n)=0=(\hat{F}-n)(\hat{F}+1)$. If $(\hat{F}+$ 1) $(\varphi)=0, \varphi \in S_{0}^{n}$, then $(\hat{F}-n)\left[-(n+1)^{-1} \varphi\right]=\varphi$, hence $\varphi \in \operatorname{im}(\hat{F}-n)$. Similarly, if $(\hat{F}-n)(\varphi)=0$, then $(\hat{F}+1)\left[(n+1)^{-1} \varphi\right]=\varphi$, hence $\varphi \in \operatorname{im}(\hat{F}$ $+1)$.

LEMMA 11. Let $\psi \in S_{0}^{n}$. Then $\psi=\hat{E}(\varphi)$ for some $\varphi \in S_{0}^{n+1}$ if and only if $\psi=\hat{F}_{j}(\psi)$ for $j=1, \ldots, n$.

Proof. First suppose $\psi=\hat{E}(\varphi)$, so that

$$
\psi\left(e_{1}, \ldots, e_{n}\right)=(n+1) \varphi\left(e_{1}, \ldots, e_{n},-e_{1}-e_{2}-\cdots-e_{n}\right) .
$$

Since $\varphi \in \operatorname{im} \sigma$.

$$
\begin{aligned}
\hat{F}_{j}\left[\psi\left(e_{1}, \ldots, e_{n}\right)\right]=\psi\left(e_{1}, \ldots, e_{j-1},-e_{1}-\ldots-e_{n}, e_{j+1}, \ldots, e_{n}\right) \\
\quad=(n+1) \varphi\left(e_{1}, \ldots, e_{j-1},-e_{1}-\ldots-e_{n}, e_{j+1}, \ldots, e_{n}, e_{j}\right) \\
\quad=(n+1) \varphi\left(e_{1}, \ldots, e_{j-1}, e_{j}, e_{j+1}, \ldots, e_{n},-e_{1}-\ldots-e_{n}\right) \\
\quad=\psi\left(e_{1}, \ldots, e_{n}\right) .
\end{aligned}
$$


Conversely, if $\hat{F}_{j}(\psi)=\psi$ for all $j$, let

$$
\varphi\left(e_{1}, \ldots, e_{n+1}\right)=(n+1)^{-2} \sum_{j=1}^{n+1} \psi\left(e_{1}, \ldots, e_{j-1}, e_{j+1}, \ldots, e_{n+1}\right) .
$$

Then

$$
\begin{aligned}
& \hat{E}\left[\varphi\left(e_{1}, \ldots, e_{n+1}\right)\right] \\
& =(n+1)^{-1}\left[\sum_{j=1}^{n} \psi\left(e_{1}, \ldots, e_{j-1}, e_{j+1}, \ldots, e_{n},-e_{1}-\cdots-e_{n}\right)\right. \\
& \left.\quad+\psi\left(e_{1}, \ldots, e_{n}\right)\right] \\
& =(n+1)^{-1}\left[\sum_{j=1}^{n} \hat{F}_{j}(\psi)+\psi\right]=\psi .
\end{aligned}
$$

COROLlary 12. The sequence

$$
S_{0}^{n+1} \stackrel{\hat{E}}{\rightarrow} S_{0}^{n} \stackrel{\hat{F}-n}{\rightarrow} S_{0}^{n}
$$

is exact.

Proof. Let $\psi \in S_{0}^{n}$. Using the previous lemma, it suffices to show that if $\hat{F}(\psi)=n \psi$ then $\hat{F}_{j}(\psi)=\psi$ for each $j=1, \ldots, n$. We have

$$
\sum_{j=1}^{n} \hat{F}_{j}(\psi)=n \psi
$$

Applying $\hat{F}_{k}$ to both sides of this equation yields

$$
\sum_{\substack{j=1 \\ j \neq k}}^{n} \hat{F}_{j}(\psi)+\psi=n \hat{F}_{k}(\psi)
$$

Subtracting the last two equations shows

$$
\hat{F}_{k}(\psi)-\psi=n \psi-n \hat{F}_{k}(\psi)
$$

which proves the result.

Piecing together the exact subsequences of Corollaries 6, 10 and 12, we deduce that for $n \geqslant 1$, the transformed sequence

$$
0 \rightarrow S_{0}^{n+1} \stackrel{\hat{D}}{\rightarrow} S_{0}^{n+1} \stackrel{\hat{E}}{\rightarrow} S_{0}^{n} \stackrel{\hat{F}-n}{\rightarrow} S_{0}^{n} \stackrel{\hat{F}+1}{\rightarrow} S_{0}^{n} \stackrel{\hat{F}-n}{\rightarrow} S_{0}^{n} \rightarrow \cdots
$$

is exact. Theorem 4 now implies the exactness of the sequence

$$
0 \rightarrow R_{n+1} \stackrel{D}{\rightarrow} R_{n+1} \stackrel{E}{\rightarrow} R_{n} \stackrel{F-n}{\rightarrow} R_{n} \stackrel{F+1}{\rightarrow} R_{n} \stackrel{F-n}{\rightarrow} R_{n} \rightarrow \cdots
$$

for $n \geqslant 1$. Combining these sequences and the trivial sequence $0 \rightarrow \mathbf{R} \rightarrow \mathbf{R}$ $\stackrel{D}{\rightarrow} 0$, and recalling how the operator $N$ acts on each $R_{n}$, completes the proof of Theorem 1. 


\section{REFERENCES}

1. I. M. Gel'fand and L. A. Dikii, Asymptotic series for Sturm-Lionville equations and the algebra of the Korteweg-deVries equation, Russian Math. Surveys 30 (1975), 63-100. (Russian)

2. H. Federer, Geometric measure theory, Springer-Verlag, New York, 1969.

Department of Mathematics, University of Chicago, Chicago, Illinois 60637

Department of Mathematics, Brown Universtty, Providence, Rhode Island 02912 